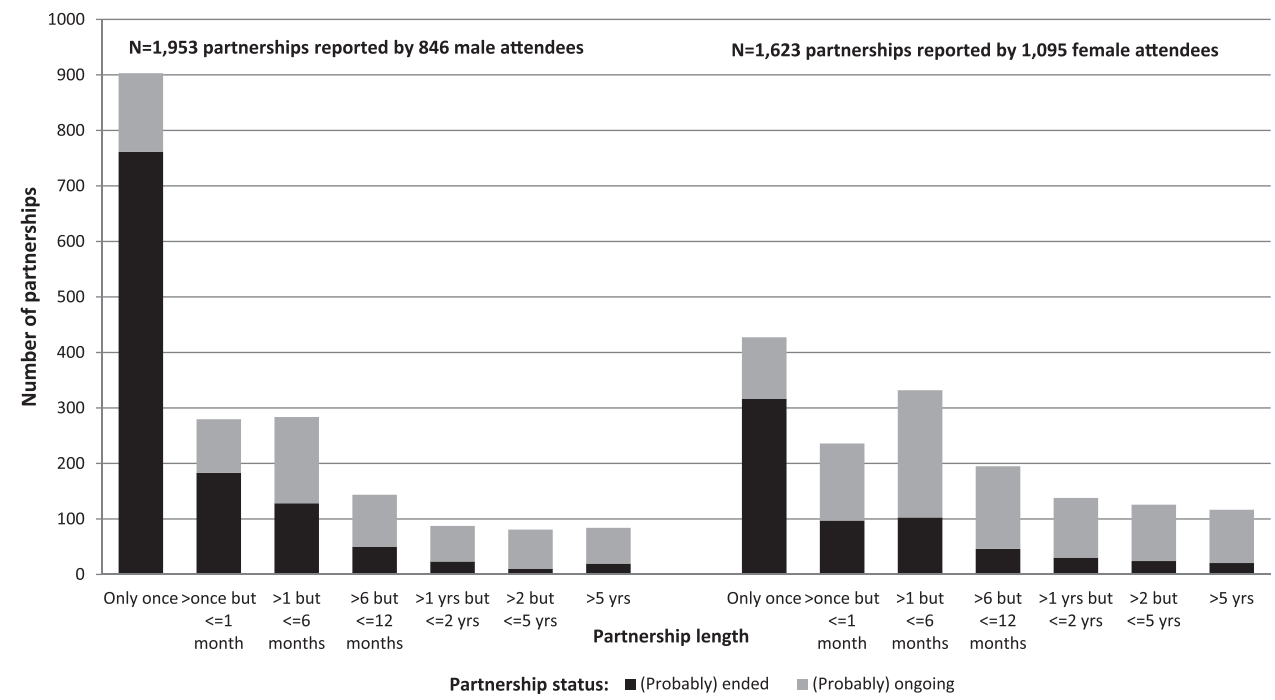

Abstract P2-S4.02 Figure 1 Distribution of partnership length by partnership status and gender of GUM clinic attendee.

Results Most patients had few partners: median: 1 partner, IORs: 1-3 (men) and 1-2 (women). However, the 1941 attendees reporting partners reported a total of 3576 partners: 1953 by 846 men, 1623 by 1095 women. Men's partnerships were shorter than women's: $73 \%$ vs $54 \%(p<0.01)$ were $<3$ months "old" (49\% vs $27 \%$, respectively, were one-off encounters), and were more likely to be considered as ended: $64 \%$ vs $41 \%$, respectively, $p<0.01$, see Abstract P2-S4.02 figure 1 Inconsistent condom use was high, especially in women's partnerships: $81 \%$ vs $65 \%$ among men's partnerships, $p=0.002$. Men (but not women) were more likely to have acute STIs diagnosed if they had never used condoms with their partners than if they had at least once: $33 \%$ vs $27 \%, p=0.001 .24 \%$ of men vs $14 \%$ of women had recent concurrent partnerships based on the dates of their 3 most recent partners $(p<0.01)$, but this was not associated with having acute STI diagnoses.

Conclusions Despite low median partner numbers, GUM attendees have a large population of partnerships, of which only a small minority involve consistent condom use, thus the potential for STI transmission is high. These partnerships are often casual in nature being short in duration and unlikely to be ongoing. Research is needed to develop and test alternative methods for reaching such partners to ensure that PN achieves both individual and public health benefit.

\section{P2-S4.03 PREVALENCE AND RISK FACTORS FOR CHLAMYDIA TRACHOMATIS INFECTION AMONG FEMALE MEDICAL STUDENTS AND WOMEN ATTENDING FAMILY PLANNING CLINICS IN NORTHERN ITALY}

doi:10.1136/sextrans-2011-050108.335

${ }^{1} \mathrm{~A}$ Biglino, ${ }^{1} \mathrm{G}$ Montrucchio, ${ }^{1} \mathrm{R}$ Moglia, ${ }^{1} \mathrm{E}$ Concialdi, ${ }^{1} \mathrm{C}$ Bolla, ${ }^{2} \mathrm{M}$ Tramontano, ${ }^{3} \mathrm{D}$ V Ferrero. ${ }^{1}$ "Cardinal Massaja" Hospital / University of Torino, Asti, Italy; ${ }^{2}$ Family Planning Clinic, A.S.L. 19, Asti, Italy: ${ }^{3}$ University of the Pacific, Stockton, USA

Background Prevalence and risk data concerning Chlamydia trachomatis CT in Italy is scare. We present a study of the prevalence and predictive risk factors for CT infection by comparing two different female populations at risk because of age (18-26) but with different socio-cultural backgrounds.

Methods An anonymous questionnaire concerning sexual behaviour was given to 493 female medical students aged 18-26 at the University of Torino and to women aged 18-26 attending three Family Planning Clinics (FPC) in Asti, Italy. A first-catch urine sample was collected into a liquid transport medium (UPT, Becton
Dickinson) from each subject who completed the questionnaire. Nucleic acid amplification testing for CT was performed using the $\mathrm{BD}$ ProbeTec ET assay. Age and behavioural characteristics were compared.

Results 6 women of 493 medical students tested (1.22\%) were positive for CT, while 11 of 262 women attending FPC had a positive CT result $(4.2 \%) \quad\left(\chi^{2}=5.7 ; p=0.017\right)$. Different behaviours between the female medical students and the FPC population respectively for non-barrier contraceptive methods were: (34\% vs $59 \%$ ); age $<18$ at first intercourse ( $40 \%$ vs $60 \%$ ); more than 1 partner during last year ( $16 \%$ vs $20 \%$ ), or the last 5 years ( $2 \%$ vs $56 \%$ ). Risk of infection was associated with $>4$ partners during the last 5 years both in medical students and to a lesser extent in women attending FPC (OR 10.7; CI 2 to 49 and, respectively (OR 3.7; CI 1.2 to 12). Age at first intercourse was also a factor. Women in FPC whose age was $<18$ at first intercourse were at higher risk of CT (OR 7.6; CI 1.1 to 30) than the female medical students (OR 1.5; CI 0.3 to 8 ). Logistic regression confirmed that $>4$ partners during the last 5 years in both groups (Wald 7.3; $p<0.01$ [students]; 3.7 $\mathrm{p}<0.05$ [FPC subjects]) and an age $<18$ at first intercourse only in FPC subjects (Wald 3.5; $\mathrm{p}<0.06$ ) were independent predictors of infection.

Conclusions Female medical students showed a significantly lower prevalence of CT compared to the female population of same age but different socioeconomic background. The number of partners in the last 5 years and age at first intercourse were the factors regarding this difference. Awareness and the level of knowledge, possibly dependent on cultural factors, seem to reduce the prevalence. The importance of targeted CT screening programs to groups at greatest risk, while promoting widespread educational efforts and awareness of risk among general population is apparent.

\section{P2-S4.04 MOTIVATIONAL INTERVIEWING IS EFFECTIVE FOR REDUCING HIGH RISK SEXUAL BEHAVIOUR}

doi:10.1136/sextrans-2011-050108.336

${ }^{1} \mathrm{~J}$ Boman, ${ }^{2} \mathrm{H}$ Lindqvist, ${ }^{3} \mathrm{~A}$ Brandell-Eklund, ${ }^{2} \mathrm{~L}$ Forsberg, ${ }^{1} \mathrm{E}$ Nylander. ${ }^{1}$ Umeå University, Umeå, Sweden; ${ }^{2}$ Karolinska Institute, Sweden; ${ }^{3}$ Swedish National Institute of Public Health, Sweden

Background Motivational interviewing (MI) can be effective for behaviour change even when tailored to brief encounters. MI is focused on resolving the ambivalence that might prevent change. 